\title{
Components and implementation of a picture archiving and communication system in a prototype application
}

This article was published in the following Dove Press journal:

Reports in Medical Imaging

\author{
Hasan H Khaleel' \\ Rahmita OK Rahmat ${ }^{2}$ \\ Dimon M Zamrin ${ }^{3}$ \\ 'Department of Medical Devices \\ Techniques' Engineering, AL-Esraa \\ University College, Baghdad, Iraq; \\ ${ }^{2}$ Department of Multimedia, Faculty \\ of Computer Science and Information \\ Technology, Universiti Putra Malaysia, \\ Serdang, Selangor, Malaysia; ${ }^{3}$ Faculty of \\ Medicine, Universiti Teknologi MARA, \\ Sungai Buloh, Selangor, Malaysia
}

Purpose: The purpose of this article was to represent the first experience of applying a picture archiving and communication system (PACS) at the Universiti Putra Malaysia with the cooperation of Universiti Teknologi MARA hospital, and to analyze the applicability of PACS, its impact on health care, its benefits to medical employees, and propose a prototype application of PACS. Methods: The main PACS components were discussed, HL7 and DICOM standards were introduced, and a prototype of WebXA application was proposed.

Results: The results of WebXA revealed the ability of this application to retrieve, store, and display angiography images on a web browser anywhere, as long as an Internet connection is provided.

Conclusion: This article presented PACS with its components and standards, a prototype application was discussed and evaluated, and a few recommendations have been provided for more improvements in the future.

Keywords: picture archiving systems, DICOM, web-based viewer

\section{Introduction}

Picture archiving and communication systems (PACS) have become one of the most popular health care systems between 2003 and 2008. ${ }^{1,2}$ During this period, archiving media and interpretation media changed from film based to digital imaging, which was considered as a big breakthrough, where digital image acquisition devices have become more famous than the classic radiology conventional systems. Therefore, once a digital image of the chest is captured, it can be processed directly by the computer. ${ }^{3}$

The digital imaging PACS is a combination of hardware and software hybrid system that is used to acquire, store, deploy and retrieve medical images using Digital Imaging and Communications in Medicine (DICOM) standard. The images and reports are transmitted digitally via PACS by integrating the system with the radiology information system (RIS) and hospital information system (HIS). ${ }^{2}$ This integration of PACS-RIS-HIS would eliminate the need to manually store, retrieve and display film jackets. Earlier, the majority of health care systems were adopting the conventional way of storing and displaying patients' data in hospitals, which delayed the time from imaging to reporting of the interpretation. Providing medical stuff with information in a short period of time is an important step in the current medical systems. Therefore, PACS is becoming a vital step and should be included in hospitals to speed up doctors' mission in curing patients.

The PACS was first embodied in mid 1970s. ${ }^{4}$ Professor Jean-Raoul introduced DIOGENE, a medical information display system at the Geneva University Hospitals in Switzerland. This system was later modernized to form PACS. Currently, PACS has
Correspondence: Hasan H Khalee Department of Medical Devices Techniques' Engineering, AL-Esraa University College, 52nd Street, Al-Karada, Baghdad, Iraq Tel +964772762 7722 Email hassan.upm@gmail.com 
been adopted in many hospitals and medical institutes. By digitizing the medical images, institutes were able to minimize costs in data management and storage and to reduce time consumption in data transportations. ${ }^{2}$ Images, as it is widely known, are the basis in teaching medical imaging. Therefore, it is vital to provide the students of medical imaging with high-quality images in order to improve the ability of analyzing images. ${ }^{5}$ Depending on this fact, a study was established to combine the current PACS with the medical imaging teaching method to design a better way of imaging teaching systems in higher institutes. ${ }^{5}$

Before PACS, the examination cycle of a radiology department usually flows in the following steps as illustrated in Figure $1:^{6}$

- a patient is directed to the ward of physicians for medical checkup;

- the responsible physician may refer the patient to the $\mathrm{X}$-ray laboratory for imaging;

- after imaging, the films will be taken manually to the reading room to be printed out and analyzed by the radiologists in the radiology department;

- the radiologist will direct the analysis of X-ray images manually to a clerk to type out the report

- the radiologist's report will manually reach the same physician as the first step to decide;
- the report might also reach outside clinics, other hospital departments, emergency room (ER) or intensive care unit (ICU).

This turnaround time of examination cycle may vary from hours to days, and this cycle might consume much time to get imaging reports which can delay the decision making about the condition of patients.

The major limitations of the conventional examination radiology cycle are as follows:

- time consuming - decision making of diagnostic results may not be obtained in a timely manner;

- high possibility of losing the examination data of patients - implying examination retake;

- physical retrieval of films from library and then from ER may take minutes to hours;

- decision making by referring physician(s) varies from hours to days;

- digitizing the films is necessary to save a copy of the images.

After PACS installation, the examination workflow is in the following steps:

- technician takes digital images in the X-ray laboratory;

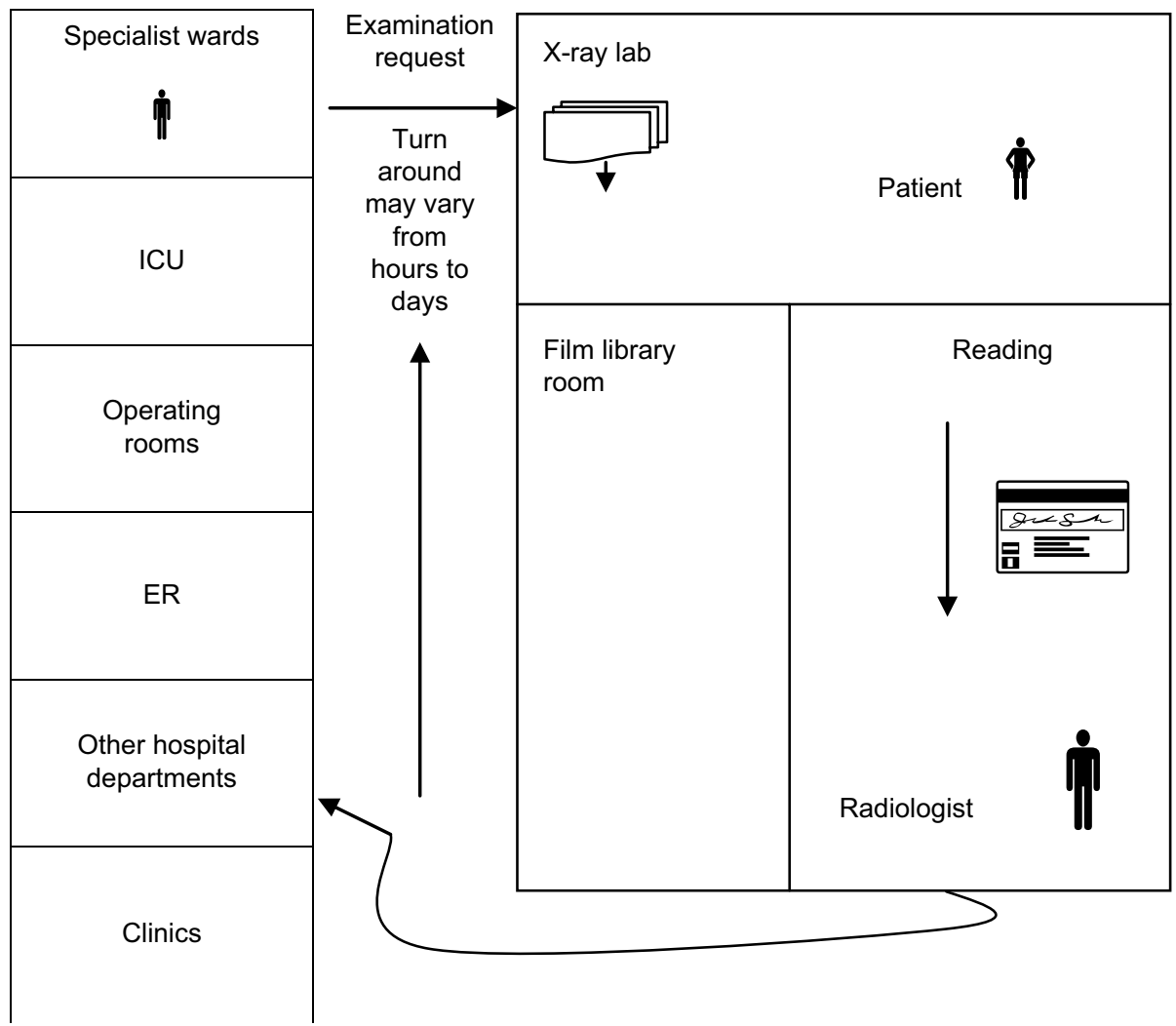

Figure I The examination cycle of a radiology department before PACS.

Abbreviations: ER, emergency room; picture archiving and communication system. 
- a few seconds later, the exposure was adjusted at the modality workstation (display workstation);

- images are then sent to digital archive;

- images are immediately available to radiologist(s), referring physician(s) office, and anywhere in the medical institute.

Therefore, with a smaller and more efficient file room, the health care is improved with more physician's satisfaction. Figure 2 briefly presents the workflow after PACS installation.

This article presents and analyzes the experience of using PACS in University Putra Malaysia (UPM) with the cooperation of UiTM hospital. Few PACS servers and DICOM viewers, such as K-PACS v1.6.0, ConQuest v1.4.17, ONIS v2.5.1.6 and ClearCanvas v7.1, have been installed and tested on local computers of the medical institutes. All these used servers are downloaded for free as trial versions for testing purposes only. The issue of database integration is an area of ongoing work at our institution. The current medical systems are mostly dependent on various systems across different departments. Our goal is to overcome the problem of various databases and integrate them in one reliable database and system that can gather all data of different departments in one database to store, deploy and display the medical data in a way that can save cost, time and effort and eliminate data duplication.

Therefore, our medical team continues research to achieve this goal. Meantime, the team managed to publish few articles related to the target of PACS. In one of the previous research works, the authors discussed problems of multisystem distribution and ways to overcome it. ${ }^{7}$ They proposed a design to integrate the medical databases to assist the medical staff in their mission. Such a design can allow easier communication between different systems via multiple platforms and languages. This step can minimize errors and risks, faster decision making, improve data management and save time and cost. In the meanwhile, a previous research proposed a conceptual database design to create a smart medical system in clinics. ${ }^{8}$ This design plays a vital role in combining the medical subsystems to make a complete system for cardiothoracic surgery unit.

Finally, a project was designed to form an integrated algorithm which integrated CAD systems with PACS using big computing infrastructure. ${ }^{9}$ This work aimed to create a system where users can request a CAD service and get the outcome to their PACS. This system helped the end users request to obtain the results in any modality.

\section{Materials and methods}

This section discusses the main PACS components followed by introduction of the HL7 and DICOM standards and the proposed prototype of WebXA application.

\section{PACS components}

PACS consists of four major components: image acquisition devices (imaging modalities), communication networks, PACS archive and server, and integrated display workstations (WS). ${ }^{8}$ PACS can be further connected to RIS and HIS health care systems via PACS communication networks as shown in Figure 3.

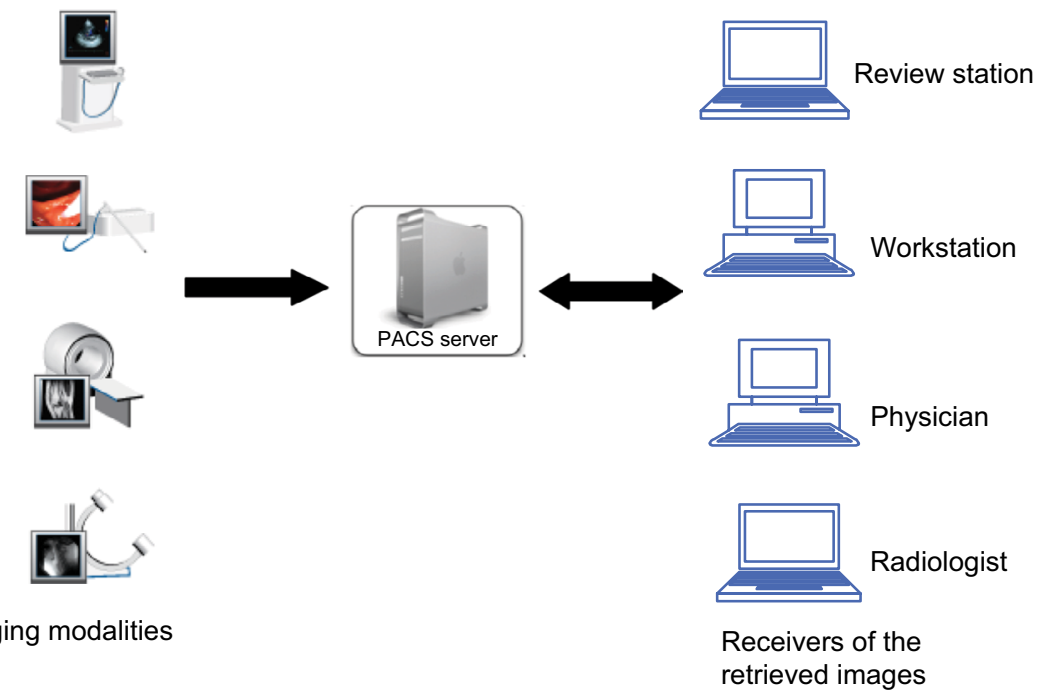

Figure 2 The examination workflow of a radiology department after PACS. Abbreviation: PACS, picture archiving and communication system. 


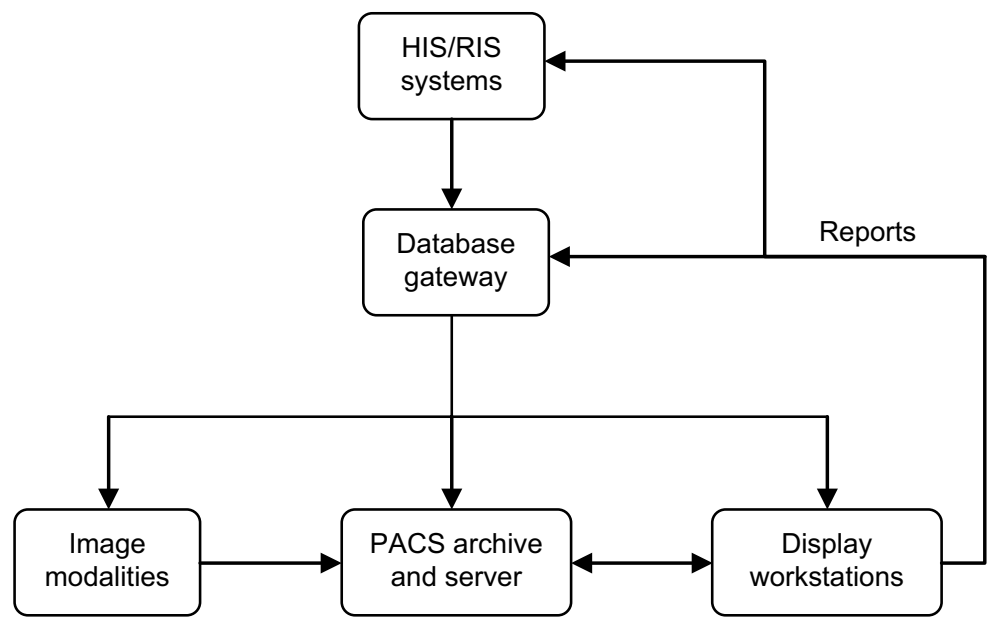

Figure 3 PACS basic components and workflow.

Abbreviations: HIS, hospital information system; PACS, picture archiving and communication system; RIS, radiology information system.

\section{Image acquisition devices}

The imaging acquisition devices are composed of the devices of imaging modalities and acquisition gateway computers. Imaging modalities include magnetic resonance imaging, computed tomography, PET, X-ray angiography, echocardiography and others. These modalities are interfaced with the PACS server via acquisition gateway computers. ${ }^{8}$ The major roles of using acquisition gateway computers are to acquire images from the imaging modalities, convert the format of the images data from manufacturer's specification to the PACS standard format, which is called DICOM, and to perform some preprocessing functions on the data such as resizing, background removal and orientation calibration. ${ }^{6}$

The two common methods of image acquisition are digitization of films and direct digital acquisition. The digitization of plain films is a vital method to convert the radiology projections (films) into digital images, because computers can process only digital images. This can be achieved using film/image digitizers such as laser scanner or charge coupled device (CCD). The second method of image acquisition is the capturing of direct digital images, which can be done using the currently developed X-ray devices. These devices can acquire digital images without the need of imaging plates used in conventional radiography. Therefore, digital images are obtained from $30 \%$ of image acquisition devices such as magnetic resonance imaging, computed tomography, ultrasound, and digital subtraction angiography.

\section{Communication networks}

The PACS communication network is the way of moving the medical data between the components of PACS themselves and other systems and to remote locations. Similar to other computer networks, PACS network provides a path to communicate between imagine modalities, gateway computers, PACS server, display and review WSs, HIS/RIS systems and any other remote medical locations. The factors of PACS networks are the network topology, line capacity and workflow assignments. The topology of communication networks refers to the physical or logical way of designing these networks, whereas two or more nodes connect to a link and then two or more links can form a network topology. The five main topologies used in the medical environments are Ring, Star, Tree, Bus and Mesh. ${ }^{6}$

Theoretically, three main types of networks are used to transfer the medical data of radiology:

- LAN network within one medical department to link imaging modalities, archive and data storage and the display WSs;

- LAN network to link different departments in a hospital (intra-hospital);

- Tele-radiology network to transfer medical data to other remote hospitals in that region.

\section{PACS Archive and server}

All the patients' information and imaging examination are sent to the PACS server for archiving. The data are sent to PACS server from the acquisition gateway computers and from HIS/RIS systems. The PACS server, which is the heart and engine of PACS, has two main components: storage media (database) and archive system. The archive system of PACS needs two levels for archiving: short term and long term. The data (images) from the short-term level are retrieved in 2 seconds, whereas those from the long-term level are retrieved in $£ 3$ minutes. ${ }^{6}$ 
Examples of the storage media used for archiving are as follows:

- (RAID) redundant array of inexpensive disks for prompt access of current images;

- magnetic plates for speedy recovery of reserved images;

- erasable magneto-optical plates for impermanent longhaul archive;

- (ROM) read only memory in the optical plate library, which constitutes the changeless document;

- recently created advanced flexible plates (DVD-ROM) for ease changeless document;

- the advanced straight tapes for reinforcement.

PACS servers have many significant functions, of which some of them are listed below:

- gets images from examinations through securing portals,

- extracts content data depicting the accepted examination from the DICOM image header;

- updates the database administration framework;

- determines the display WSs to which recently created examinations are to be sent;

- automatically recovers important correlation images from recorded examinations;

- store stockpiling or long-haul library file framework;

- automatically revises the introduction of registered or advanced radiography pictures;

- determines ideal complexity and brightness parameters for displaying images.

\section{Display WSs}

The display WS is a very important component of the PACS network, which plays a vital role in the clinical acceptance of PACS. It is the hardware component that replaces the Alternator or the manual light box of radiology system. Today, most radiologists analyze films in a perusing room utilizing light boxes or alternators. The light boxes are lighted boards on which $\sim 12$ films may be hung at once for review and physically turn about 8 of 200 films of a patient into position for diagnosing purposes. Simple image preparing operations such as zooming using an amplifying glass and annotation of films are performed utilizing the alternators. Display WSs help radiologists make primary diagnosis and hence they are also named as diagnostic WSs. These WSs are composed from local storage database, network connection for communications, resource management, display, and processing software. Display WSs provide some of the basic image processing functions such as access, manipulation, evaluation, and documentation.

\section{HL7 and DICOM standards}

Transmission of images and reports between different medical institutes is a hard mission for two reasons: first, information systems utilize various machine platforms, and second, the medical images and information are created from different imaging modalities by distinctive producers. ${ }^{8}$ With the growing medical standards, Health Level 7 (HL7) and DICOM, incorporation of heterogeneous, different restorative images and information into a composed system is made feasible. Interfacing two medical systems requires two elements: a normal data format and a correspondence protocol. HL7 is a standard text-based information format, and DICOM incorporates data format and correspondence protocols. ${ }^{10}$ In compliance with the HL7 standard, DICOM is conceivable to exchange medicinal information such as HIS, RIS and PACS. By adjusting the DICOM standard, the medical images created from an assortment of modalities and manufacturers might be interfaced as an incorporated health care system.

HL7, introduced in March 1987, was sorted out by a client-vendor board to create a standard for electronic information trade in health care environments, particularly for hospital provisions. HL7 standard alludes to the highest level, the provision level, in the seven communication levels of Open Systems Interconnection (OSI). The main objective is to improve the interface execution between $\mathrm{PC}$ provisions from different manufacturers. This standard confirms exchanging data among health care systems, for example, HIS, RIS and PACS. On the other hand, DICOM is a significant standard which has been developed as a consequence of the starting exertions by ACR and NEMA joint council shaped in 1993 to push correspondence of computerized image data regardless of gadget producer. This standard encourages the advancement and development of PACS to interface with different systems of healing center data in a similar way. In addition, DICOM permits the making of indicative databases that could be cross-examined by a wide assortment of gadgets conveyed geologically.

A work in 2008 discussed the integration of a research called content-based image retrieval with RIS and PACS. ${ }^{11}$ This work aimed to improve the workflow of radiological daily routine. The importance of this integration comes from making all the PACS archive available for radiologists to find an accurate diagnosis for the current study of patients. In this article, the integration between RIS and PACS is achieved in WebXA where all patients' studies (images and information) are saved in the database of the server, and WebXA can call any study using the name of the patient and number of the 
study. Specialist can then illustrate the images of a specific study and analyses for better diagnosing from the current study.

ACR-NEMA, officially known as the American College of Radiology and the National Electrical Manufacturers Association, established a committee to create a set of benchmarks to serve as a common background for different therapeutic medical imaging vendors. The main objective was that recently created instruments have the capacity to impart and partake in imparting therapeutic image data, specifically, inside PACS domain. The committee, which focused primarily on issues concerning data exchange, interconnectivity, and communications among the health care systems, started work in $1982 .^{6}$

\section{WebXA prototype application}

WebXA is the PACS prototype application which has the same structural design of client-server except that the software for the client and server is Web-based application. Extra advantages of Web-based design server over the client-server are as follows: first, the customer WS equipment might be hardware independent as long as the Web software is underpinned. Second, the Web-based software is totally movable, ie, the Web-based application might be utilized on any location as long as an Internet connection is provided. The disadvantage of Web-based application is that its functionality and performance are limited compared to the client server. One of the most important purposes of using Web-based viewers and applications in health care systems is the real-time telecommunication or teleconsultation. However, what is the teleconsultation process? and is there any application(s) to support it? In this paper, we explained this process and tested a Web-based prototype application to support the teleconsultation tool.

The teleconsultation is addressing different scenarios in the health care institutes. Let us consider the following scenario: when a doctor in the physician department wants to consult with a radiologist in the reading room about a written report of a patient in the checking room. This consultation process should occur without leaving, both specialists, their wards and walk all the way to each other's departments. Therefore, the teleconsultation is a circumstance in which two or more specialists located in various departments need to discuss and consult about a patient's results without leaving their departments. Earlier, there were few results of teleconsultation tools, such as Televideo, NetMeeting which is a sharing software from Microsoft, Proshare from Intel, and PCAnywhere from Symantec. However, most of these applications lack for convincing applicability of image processing functions which are becoming necessary in the health care environments.

In this paper, a Web-based viewer application, called WebXA, has been created and tested on the PACS. This application is established for viewing and primary diagnostic purposes only where the medical imaging software is integrated with the communication facilities to develop a remote viewing and consultation tool using the TCP/IP protocol.

\section{Results}

WebXA is to retrieve, store and display the angiography images on a web browser anywhere as long as an Internet connection is provided. A real-time text exchanging property is added to WebXA application to make it easy for specialists to exchange comments and consult about patients' reports and results, as shown in Figure 4. WebXA viewer provides:

- Easy and friendly GUI contains buttons to stop the moving frames of angiography and to move forward or backward.

- Text exchange view that can be used by the specialists to exchange comments about the medical images or the primary diagnostic of patients' data.

- Client/PACS server communications via TCP/IP.

- Software functions such as save, open and print.

Few open-source PACS servers and DICOM viewers, such as K-PACS v1.6.0, ConQuest v1.4.17, ONIS v2.5.1.6 and ClearCanvas v7.1, have been used in this study to test the workflow of PACS components. . A prototype application for DICOM viewing and teleconsultation purposes application has been developed in this study, where this application (WebXA) can store, display, download, send and print the angiography images via Internet explorers. Since exchanging information of patients among specialists who are located in different geographically area is a vital process, a comment box has been added to WebXA viewer to give the ability to medical doctors and radiologists to discuss medical reports and results of patients.

The proposed WebXA application mimics the functionality of PACS discussed above where it stores, retrieves and illustrates coronary angiography images for radiologists and surgeons directly on their mobile phones anytime and anywhere in order to save time and efforts of diagnosing. This is a very important step for the medical doctors. The application is tested with its initial/preliminary results by a heart surgeon, and he was greatly satisfied for being able to use his personal mobile phone to check his patients' data anywhere and anytime in the hospital or even outside. 


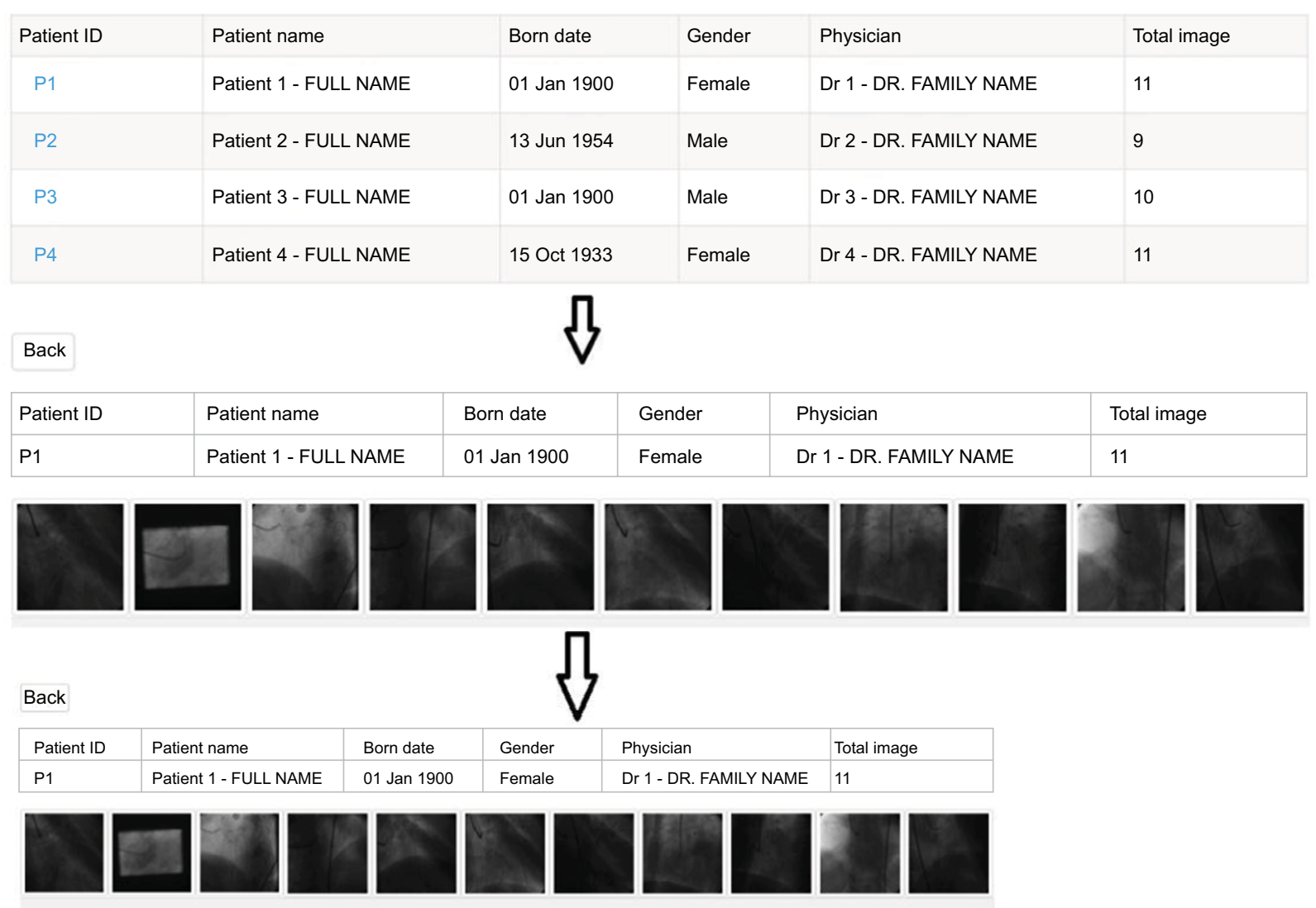

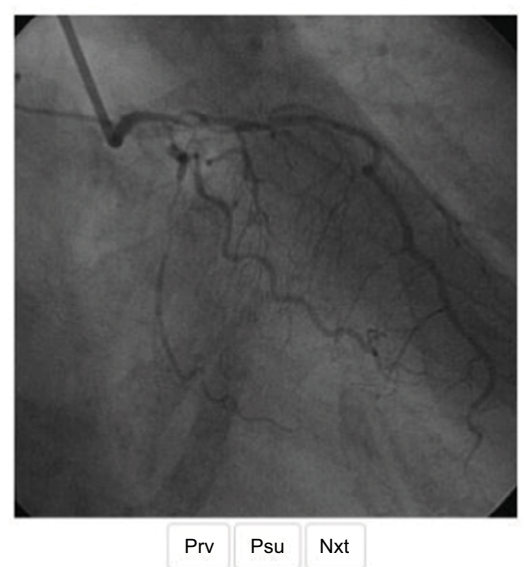

Exchange comments

Figure 4 Web-based angiography viewer WebXA.

Note: The patient and physician details in the figure are examples formats only.

Abbreviations: Nxt, next; Prv, previous; Psu, pause.

\section{Discussion}

PACS, its components, network and benefits have been discussed in this paper. This system is an electronic and preferably filmless data system for obtaining, sorting, transporting, putting away and electronically displaying health care images and data. ${ }^{12}$ Digital imaging vendors push this system profits, counting the end of unreasonable silverbased film, progressed access to new and old movies for all clinicians, lessening in the physical stockpiling prerequisite of massive movies, and more level work force costs. In this paper, a restricted client study was attempted at the Hospital Sungai Buloh, Malaysia, in cooperation with the UPM preceding the advancement of the web program engineering with numerous fewer clients having admittance to the system. The study and implementation focused on restorative staff and on the accessibility of medical images and reports prior and then afterward the PACS establishment. The present study focuses on the effect that PACS has made to the working of 
the clinic overall and on the living up to expectations lives of distinct clinicians in numerous distinctive orders.

This prototype can illustrate the medical images (coronary angiography) on a mobile phone by physicians anywhere which is a very important step for the medical doctors. The WebXA was tested by the third co-author of this article (Professor Dr DM Zamrin), and he is a heart surgeon who works in the university hospital and was selected to do our tests. He was very happy with the initial results as we promised to improve the work in the future for a bigger and more accurate PACS for different types of medical images in the hospital.

Generally, PACS tries to save time by saving and distributing medical data of different modalities for radiologists to analyses. This prototype is trying to integrate patients' studies in one database and illustrate them to radiologists and surgeons in real-time. By doing this, surgeons will be able to illustrate their patient's data anytime and anywhere inside or outside hospitals which could save time for patients. The limitation of this work is that it was applied on coronary angiography only and further image modalities should be added in the future.

\section{Conclusion}

This paper presents the PACS, its components, the communication standards in PACS networks and a prototype Web-based application. The paper also discussed the conventional way of acquiring images of patients and exchanging the results among specialists (before PACS). Then after, the presentation moves to the era after PACS and the forward jump in the health care systems that PACS achieved. To prove the point of applying PACS in medical institutes, we implemented a prototype application (WebXA) for angiography display and exchange between different geographical locations. This viewer is able to display angiography and to exchange comments between specialists who are located in different departments. In addition to this application, few PACS servers have been tested with our viewer to send and receive images. The primary results of this application and tests of PACS servers are convinced and encouraging for more improvements.

\section{Acknowledgment}

The authors would like to present their appreciation for the Faculty of Medicine at Universiti Teknologi MARA, Malaysia, for their support to apply WebXA application and for their will to extend the work in the near future.

\section{Disclosure}

The authors report no conflicts of interest in this work.

\section{References}

1. Chimmanee S, Patpituck P. Picture archiving and communication system (PACS) characteristic on wired-line and wireless network for traffic simulation. In: International Conference on Information Networking 2013 (ICOIN), Jan 28, 2013: 589-594; Bangkok, Thailand: IEEE

2. Maggy T, Jacob G, Mary ER, Andrea S. Picture Archiving and Communication Systems: a 2000-2008 Study. IL, USA: Research Provided by The Dorenfest Institute for Health Information, through the HIMSS Foundation Chicago; 2012.

3. Souto M, Malagari KS, Tucker D, et al. Digital radiography of the chest: state of the art. Eur Radiol. 1994;4(4):281-297.

4. Hadžiahmetović Z, Hadžiahmetović M. Implementation of the system for image archiving and communication (PACS) at the clinical center university of Sarajevo. Medical J. 2012;19(3):251-254.

5. Dong P, Gao ZQ, Li YW, et al. The construction of medical imaging network teaching system with the PACS in university. In: 2012 International Conference on Systems and Informatics (ICSAI2012); 2012:1023-1025; Yantai, China.

6. Francis B. PACS Components, Standards and Their Implementation in a Prototype Application [thesis]. Zhejiang: Zhejiang University; 2012.

7. Murad MAA, Jabar MA, Sidi F, et al. Integration of heterogeneous databases for medical experts. In: 2012 International Conference on Advanced Computer Science Applications and Technologies (ACSAT); 2012:458-461; Kuala Lumpur, Malaysia.

8. Sani NFM, Affendey LS, Rahmat RWO, et al. Conceptual database design for smart medical clinical system. In: 2012 International Conference on Advanced Computer Science Applications and Technologies (ACSAT), 2012:472-475; Kuala Lumpur, Malaysia.

9. Suárez-Cuenca JJ, Tilve A, López R, Ferro G, Quiles J, Souto M. Integrating CAD modules in a PACS environment using a wide computing infrastructure. Int J Comput Assist Radiol Surg. 2017;12(4):657-667.

10. Huang HK. PACS and imaging informatics: basic principles and applications. Hoboken: John Wiley \& Sons; 2012;39:1681-1681.

11. Fischer B, Deserno TM, Ott B, et al. Integration of a research CBIR system with RIS and PACS for radiological routine. In: Katherine P. Andriole KP, Siddiqui KM, editors. Medical Imaging 2008: PACS and Imaging Informatics. Proceedings of SPIE. Bellingham: SPIE; 2008:6919.

12. Jorwekar GJ, Dandekar KN, Baviskar PK. Picture archiving and communication system (PACS): clinician's perspective about filmless imaging. Ind J Surgery. 2013:1-4.
Reports in Medical Imaging

\section{Publish your work in this journal}

Reports in Medical Imaging is an international, peer-reviewed, open access journal publishing original research, reports, reviews and commentaries on all areas of medical imaging. The manuscript management system is completely online and includes a very quick and fair peer-review system, which is all easy to use.

\section{Dovepress}

Visit http://www.dovepress.com/testimonials.php to read real quotes from published authors. 